As anxiety about the capacity crunch mounts, it is natural to question the best way to overcome it.

To Richardson, the best way is through better device integration of transmitters, receivers, amplifiers and reconfigurable optical add-drop multiplexers, and by exploiting these aspects of SDM with multi-parallel SMF systems to reduce the cost per bit.

"Migration to SDM systems themselves will likely take longer, and may ultimately not prove commercially attractive and viable. In addition, using increased caching of data around the network to minimize the need for long-haul transport and ultimately the adoption of different business models that discourage profligate use of capacity will also likely play a significant role," Richardson added.

To Kilper, focusing on the efficiency of the communication systems is the way to go. Footprint, thermal density, and system efficiency in general are often closely tied to energy efficiency, so energy efficiency is a good place to look for targets. According to him, to get exponential scaling from the communication systems, as we've enjoyed in the past, we need to be thinking long term about core network systems that operate at the level of $10 \mathrm{pJ} \mathrm{bit}^{-1}-$ more than 2 orders of magnitude more efficient than today - and $100 \mathrm{fJ} \mathrm{bit}^{-1}$ for on-chip input/output systems.

"Parallel growth can scale well as long as the efficiency improves in proportion to the performance, such as speed and capacity improvement. Both wireless and optical systems need to improve in efficiency at exponential rates to keep up with traffic growth. This is a daunting challenge, something that hasn't been done before, but it is technologically achievable with smart investments in research," urged Kilper.
In spite of the potentially alarming situation, both Richardson and Kilper are rather unruffled, staying optimistic.

"No need to panic. The Internet is not going to grind to a halt tomorrow. We are in an early phase of research looking to identify future options and to explore just what is possible by migrating away from current systems based on single-mode fibre and current device technology. Once the technical options are understood, the whole issue of commercial viability, cost and power reduction will need more focused consideration," said Richardson.

"This is an exciting time, with many changes coming and a convergence of multiple technologies. It should be a fun ride," said Kilper.

Rachel Won is at Nature Photonics, The Macmillan Building, 4 Crinan Street, London N1 9XW, UK. e-mail:r.won@nature.com

\title{
MICRO-OPTICS
}

\section{Light robots}

Microscale robots have been proposed for future applications including drug delivery and microsurgery. Ideally, such devices would be powered wirelessly. Several avenues for achieving this have been explored, including van der Waals and capillary effects as well as artificial muscles. However, achieving microscale miniaturization together with generating sufficient forces for interesting mobility is challenging.

Now, Hao Zeng and colleagues in Italy and Poland have reported lightdriven microscopic 'walkers' (Adv. Mater. http://doi.org/f2747b; 2015). The device, consisting of a $60 \times 30 \times 10 \mu \mathrm{m}^{3}$ main body and four conical legs about $10 \mu \mathrm{m}$ long, can jump as far as $1 \mathrm{~cm}$.

The team showed that a liquid crystalline elastomer can contract by $20 \%$ when heated to about $100^{\circ} \mathrm{C}$ by a $532 \mathrm{~nm}$ laser - this can be used to drive the appropriately designed legs of the device. Interestingly, this driving can be done at a frequency of up to $1.8 \mathrm{kHz}$.

Diederik Wiersma, one of the manuscript's corresponding authors, explained to Nature Photonics that the system can be regarded as a 'robot' on a micrometre length scale with the advantage of obtaining its energy simply by absorbing light from its environment. "Energy management is a difficult issue for

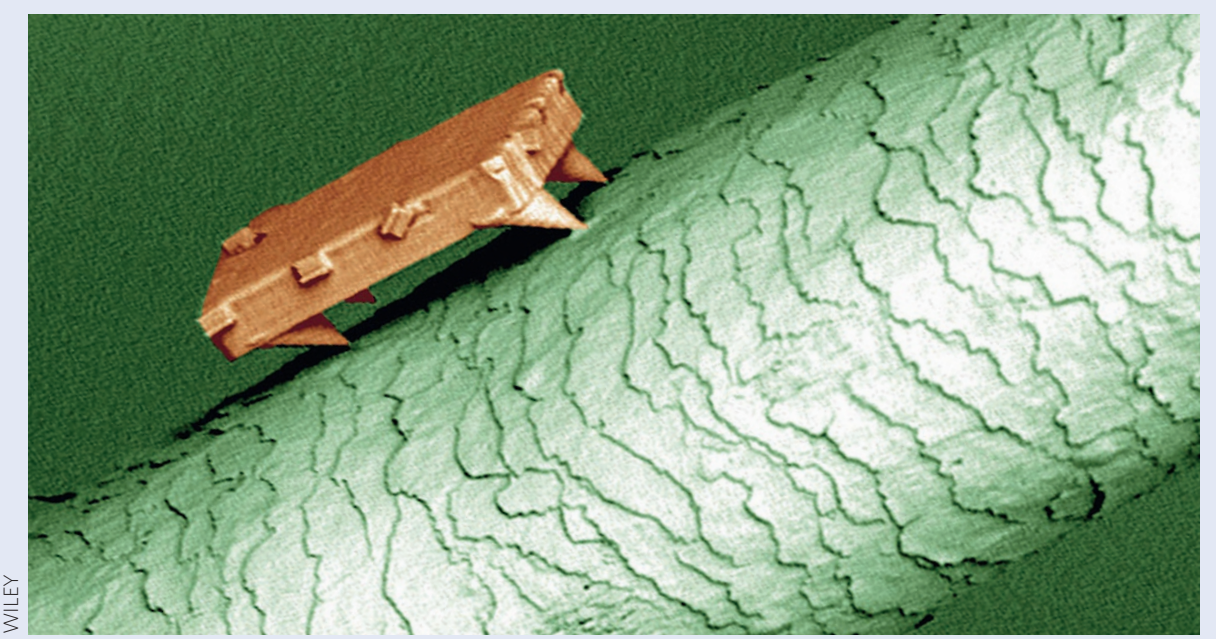

microscopic robots, as there are no batteries available that are so small," Wiersma told Nature Photonics. "Light energy solves this issue. The robot can walk, rotate, and jump and constitutes the smallest walking artificial creature, even smaller than the smallest known terrestrial living animal."

Wiersma noted that a team of chemists and physicists worked together on this for nearly five years. The work involves materials science and optics and required working out how to enable such a small-scale device to walk. "Physics becomes very different when you go to the micrometre scale. For instance, adhesion becomes immense and the robot has, literally, to walk on the tips of its 'toes'," Wiersma explained.

The idea allows the study of forces at surfaces and locomotion on the microscale but there is an additional interesting aspect, as noted by Wiersma. The microrobots are not only driven by the light, but they also influence the optical properties of their environment, opening up several areas of investigation, especially if other optical structures are integrated.

DAVID PILE 